\title{
Laparoscopic cholecystectomy delayed by complicated myocardial infarction with papillary muscle rupture, and performed after unique complex mitral repair
}

\author{
Miroslaw Bitner', Ryszard Jaszewski', Slawomir Jander ${ }^{1}$, Marek Maciejewski \\ ${ }^{1}$ Cardiac Surgery Department, Medical University of Lodz, Poland \\ 2Department of Cardiology, Medical University of Lodz, Poland
}

Videosurgery Miniinv 2013; 8 (2): 170-173

DOI: $10.5114 /$ wiitm.2011.32823

\begin{abstract}
A 65-year-old woman was admitted for laparoscopic cholecystectomy, a method of choice for gallbladder diseases. Symptoms of gallstones are similar to angina pectoris, especially in right coronary artery stenosis. In this case, masked by known symptomatic gallstones, unsuspected coronary artery disease manifested with complicated myocardial infarction and pulmonary edema. The patient survived the acute period, treated pharmacologically. Severe mitral insufficiency caused mainly by ruptured papillary muscle, with left ventricle and atrium enlargement, and right coronary artery stenosis were indications for heart surgery. Repair of this infrequent complication of myocardial infarction is rarely feasible. The complex repair, unique for this cause, is described. During the operation, the head of the ruptured posteromedial papillary muscle was re-implanted, and two neo-chords implanted for prolapsing the A2 mitral valve segment. Annuloplasty with a $29 \mathrm{~mm}$ elastic ring accomplished repair. Saphenous bypass graft was applied to the only feasible postero-lateral branch. Although intraoperative echocardiography revealed excellent results, inotropic support, and intra-aortic counterpulsation were necessary for weaning off cardio-pulmonary bypass and low cardiac output treatment. The patient was discharged home on postoperative day 12, with anticoagulant administered for 3 months. As soon as it was no longer required, she underwent laparoscopic cholecystectomy, with no complications. Durable results of both operations performed 5 years ago are confirmed by physical examination and ultrasonography. Complex mitral valve repair, rather than valve replacement, should be considered in similar cases. Possibility of coexistence of coronary artery disease should be considered before cholecystectomy. Good quality repair of cardiac disease allows for laparoscopic cholecystectomy.
\end{abstract}

Key words: laparoscopic cholecystectomy, post-infarction cardiac rupture, papillary muscles, mitral regurgitation, mitral valve annuloplasty, expanded polytetrafluoroethylene.

\section{Introduction}

Symptoms of gallstones are similar to angina pectoris, especially in right coronary artery stenosis. Coronary artery disease, and later acute coronary syndrome, in this patient were masked by known symptomatic gallstones, and diagnosed only after complicated ST elevation myocardial infarction (STEMI) occurred. Acute post-infarction mitral regurgitation (MR) caused by papillary muscle (PM) rupture occurs with a frequency of up to $3 \%$ of STEMI cases. It is a life-threatening complication, with early mortality reaching $80 \%$ without 
surgery, and still high after the surgery [1-3]. Therefore surgical reports are scarce [1, 2, 4-9], particularly on successful mitral valve (MV) repair $[2,8]$.

Laparoscopic cholecystectomy is nowadays a gold standard in gallbladder surgery, although not totally free from complications, or contraindications [10, 11].

An obvious contraindication is significant heart disease, as for any major elective surgery, except the treatment of the heart itself. The atypical course of the disease and the solution unique for this cause complex MV repair with muscle head reimplantation using polytetrafluoroethylene (PTFE) suture, artificial chords and ring implantation - are described.

This eventually allowed for laparoscopic cholecystectomy after 3-month administration of anticoagulant was completed.

\section{Case report}

In July 2007, 65-year-old woman was admitted for the operation, in NYHA functional class III, with cardiac cachexia $\left(45 \mathrm{~kg}, 155 \mathrm{~cm}, \mathrm{BSA}=1.4 \mathrm{~m}^{2}\right)$, signs of previous inferior STEMI, on sinus rhythm $85 / \mathrm{min}$, treated with diuretic, angiotensin-converting-enzyme inhibitor (ACEI), $\beta$-blocker, statin, and acetylsalicylic acid (ASA). She suffered from severe MR and coronary artery disease (CAD). Admitted 4.5 months earlier to the local hospital for an elective laparoscopic cholecystectomy for symptomatic gallstones, with a history of arterial hypertension and hyper-cholesterolemia, but without knowledge of CAD, the patient developed acute heart failure with pulmonary edema before the operation. She was treat- ed conservatively in a local internal medicine ward for an inferior STEMI with severe MR. Coronary angiography (in May 2007) revealed 75\% stenosis of the dominating right coronary artery (RCA) at the crux, and amputated acute marginal (AcM) branch. Transthoracic (TTE) and transoesophageal (TOE) echocardiography findings were as follows: enlarged left ventricle and atrium dimensions (LV 6.22/3.6 cm, LA $5.25 / 4.8 \mathrm{~cm}$ ), severe MR caused mainly by ruptured posteromedial PM (Photo 1), with prolapsing A3, P3, and also A2 mitral valve segments. Contractility evaluation revealed postero-infero-lateral wall akinesis/hypokinesis, with global left ventricular ejection fraction seemingly normal due to severe $M R$ $(\mathrm{EF}=63 \%)$. Estimated Logistic EuroSCORE was 4.6\%, and STS mortality risk equal to $3.6 \%$.

\section{Operation details (10th July 2007)}

Access via median sternotomy. Cardio-pulmonary bypass (CPB) with upper ascending aorta and bicaval cannulation was established with general normothermia and cold crystalloid antegrade intermittent cardioplegia. Distal anastomosis of saphenous bypass graft to postero-lateral ramus of the right coronary artery (the only feasible branch) was performed first. Mitral valve examination via left atriotomy parallel to the interatrial groove confirmed severe MR caused by: 1) total rupture of posteromedial PM (Photo 1) with chords leading to prolapsing $A 3$ and P3 segments and posteromedial commissural area, 2) prolapsing $A 2$ segment due to elongated chords, 3) dilation of mitral annulus.
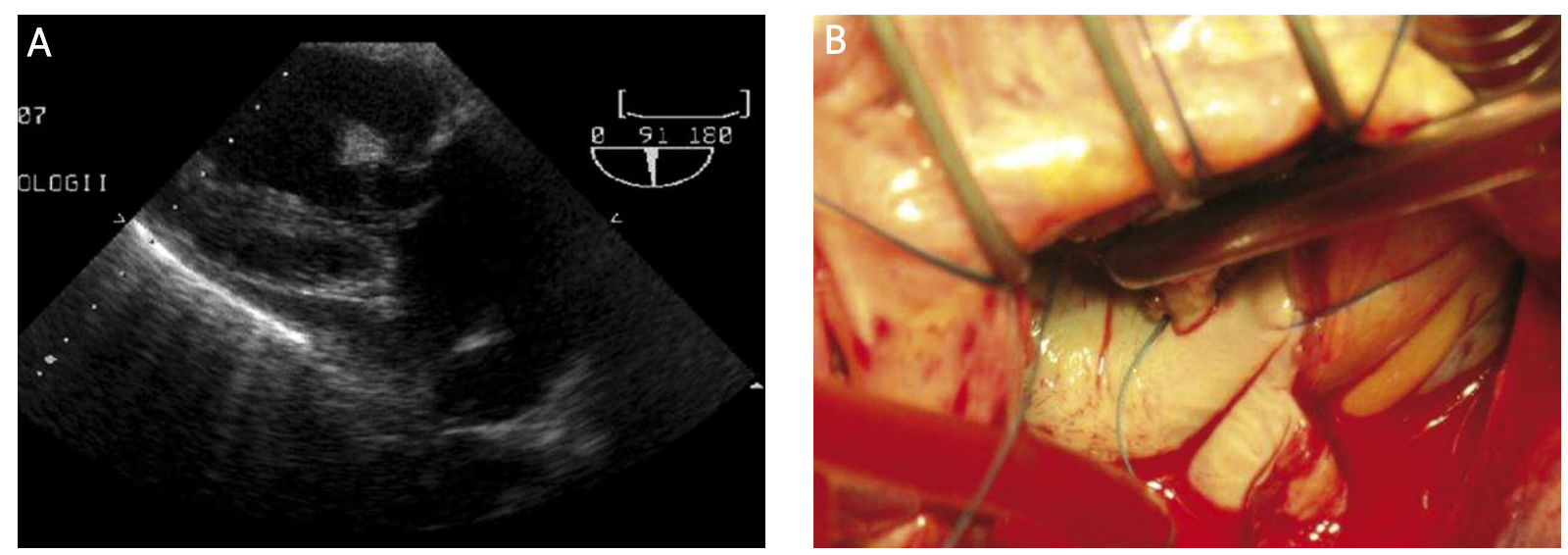

Photo 1 A, B. Ruptured head of posteromedial papillary muscle on transthoracic echocardiography, brought into view in the operating field 


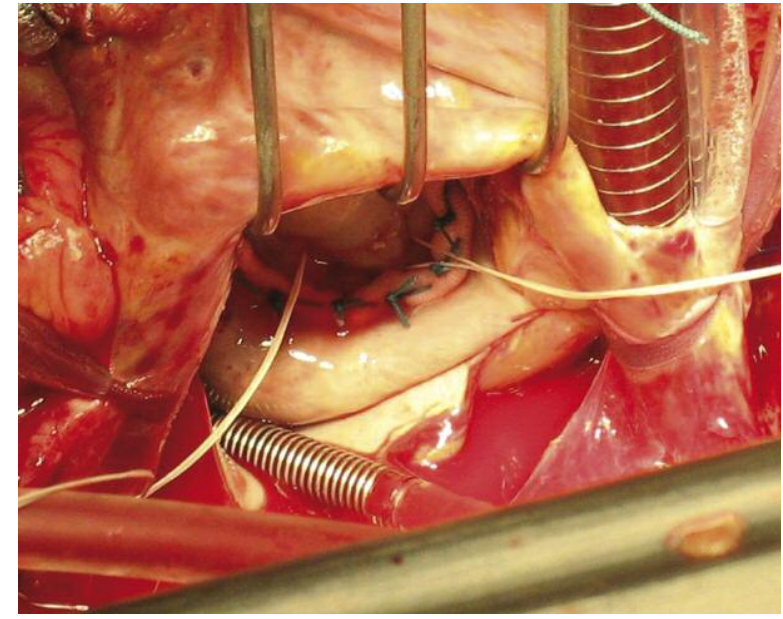

Photo 2. Extension of PTFE suture used for head re-implantation served as neo-chord for inferior part of A2 segment. Its superior part required a second chord (a view before neo-chord length was established)

The location from which the PM head broke off was invisible after that time, and the place for reimplantation was chosen so as to achieve the proper plane and area of A3/P3 cusp segments coaptation. The head was reattached with double buttressed expanded PTFE CV4 suture (W. L. Gore and Assoc, Flagstaff, AZ). The extension of this suture was used simultaneously as the neo-chord for the inferior part of the A2 segment of the anterior mitral cusp. Implantation of the second CV 4 Gore-Tex chord for anchoring the superior part of the A2 segment, and a $29 \mathrm{~mm}$ elastic ring (Duran AnCore, Medtronic) was performed next (Photo 2).

Finally, the neo-chord lengths were established. The saline hydrostatic test revealed very good results. The atrium was closed, the heart de-aired, the aorta unclamped, and the heart defibrillated. Proximal bypass anastomosis was completed on the side aortic clamp.

Intraoperative TOE revealed only a trace of residual MR. However, intra-aortic balloon pump (IABP) introduced by puncture of the right common femoral artery was necessary for weaning off CPB. Intra-aortic balloon pump was continued for low cardiac output treatment together with mechanical continuous ventilation (MCV), as well as adrenalin infusion until postoperative day (POD) 2, and dopamine infusion until POD 5. Maximal CK-MB level was $51 \mathrm{U} / \mathrm{l}$.

Transthoracic echocardiography on POD 8 revealed normalized cardiac dimensions (LV 5.2/3.12 cm,
LA 4.6/4.1 cm), local contractility improvement with normal global LV function (EF 63\%), trace of MR, and very good coaptation of mitral leaflets. The patient was discharged home on POD 12 with the sinus rhythm 64 beats/min.

Anticoagulation therapy was administered for the next 3 months. After it was completed, the patient underwent laparoscopic cholecystectomy. Good and durable results of $M V$ repair were confirmed by the physical examination that took place every 6 months, and annual echocardiography (trivial MR after 55 months). The cholecystectomy results are also good.

\section{Discussion}

Luckily the patient developed acute coronary syndrome before, not during cholecystectomy, survived acute heart failure, recovered, and was operated on electively. Moreover, in this case mitral repair was possible, which is extremely rare in such cases.

The use of neo-chords was crucial. The authors have not found in the medical literature any other case in which PTFE suture and neo-chord implantation was used for PM rupture repair.

Only a tiny number of patients with PM rupture have undergone MV repair [2, 4, 5, 8, 9]. In the patients operated on between 1980 and 2000, 51 suffering from total, and 3 from partial PM rupture, 41 of them underwent mitral valve replacement (MVR), and only $13 \mathrm{MV}$ repair (1 patient in 25 before the year 1990, and 12 in 29 after that) [2]. It is surprising that in none of the cases was neo-chord used, considering that PTFE chords have been implanted in humans since 1989/1990 [12]. The operative mortality decreased with time from $28 \%$ to $10 \%$ in the whole group. In the repaired valve group the mortality was lowered to $7.7 \%$ [2]. In the subgroup not suitable for coronary artery bypass graft (CABG) the mortality was much higher. Ninety percent of patients presented with cardiogenic shock and/or pulmonary edema and/or cardiac arrest. Postoperatively 74\% patients required IABP, inotropic support, and MCV [2]. Repair improved outcome, although there was no statistical significance for the small number.

In a multicenter study of emergently operated patients (between the years of 1986 and 2007) only 30 out of 126 (including 66 with PM rupture) of those with STEMI underwent MV repair. Only 1 patient was treated with artificial chord implantation, but there was no case of PM rupture repair reported [7]. 
Others described 42 patients with MV repaired with Carpentier's technique for post-infarction leaflet prolapse, including only one case of total PM rupture (unfortunately fatal) [5].

Despite longer time needed for the MV repair, haemodynamic results are much better than those of $M V R$, and therefore an increase in repair number should improve the survival rate. However, in urgent situations, repair could be impossible due to weakness of the myocardium. The recent meta-analysis by Vassileva et al. of mitral repair versus replacement results in ischemic mitral regurgitation confirmed better short and long term survival in the repair group [9]. However, this study excluded papillary muscle rupture cases.

Complex MV repair with myocardial revascularization in our patient brought very good results. After initial low cardiac output, the recovery was quick. The discharge on POD 12 was shorter than in similar cases operated on with MVR (for example, 71- and 34-year-old patients who underwent the operation on the $18^{\text {th }}$ and $3^{\text {rd }}$ day respectively after myocardial infarction were discharged on POD 52 and POD 42) $[13,14]$.

Nevertheless, it seems that both EuroSCORE and STS underestimate the risk for these patients. Postinfarction ventricular septal defect, giving similarly severe hemodynamic alterations, has an additional risk entry in EuroSCORE, unlike PM rupture, which surely should have one.

After the mitral repair, anticoagulant is required only for 3 months. Elective abdominal surgery was further delayed for this period. Previous successful cardiac surgery was not considered as a contraindication for laparoscopic surgery, so the patient underwent laparoscopic cholecystectomy with no complications.

\section{Conclusions}

Mitral valve repair with durable, good outcome is possible in selected cases of post-infarction papillary muscle rupture. Repair seems to have better results than valve replacement. Therefore it should be performed whenever viable. Revascularization is mandatory, if feasible. Possibility of coexistence of CAD should be considered before cholecystectomy. Laparoscopic cholecystectomy is possible after successful heart surgery.

\section{References}

1. Kishon Y, Oh J, Schaff H, et al. Mitral valve operation in postinfarction rupture of a papillary muscle: immediate results and long-term follow-up of 22 patients: Mayo Clin Proc 1992; 67: 1023-30.

2. Russo A, Suri RM, Grigioni F, et al. Clinical outcome after surgical correction of mitral regurgitation due to papillary muscle rupture. Circulation 2008; 118: 1528-34.

3. Thompson CR, Buller CE, Sleeper LA, et al. Cardiogenic shock due to acute severe mitral regurgitation complicating acute myocardial infarction: a report from the SHOCK Trial Registry: should we emergently revascularize occluded coronaries in cardiogenic shock? J Am Coll Cardiol 2000; 36: 1104-9.

4. Chevalier PH, Burri H, Fahrat F, et al. Perioperative outcome and long-term survival of surgery for acute post- infarction mitral regurgitation. Eur J Cardiothorac Surg 2004; 26: 330-5.

5. Jouan J, Tapia M, Cook RC, et al. Ischemic mitral valve prolapse: mechanisms and implications for valve repair. Eur J Cardiothorac Surg 2004; 26: 1112-7.

6. Oxorn D, Curtis W, Burnett R. Ruptured papillary muscle. Eur J Cardiothorac Surg 2000; 18: 607.

7. Lorusso R, Gelsomino S, De Cicco G, et al. Mitral valve surgery in emergency for severe acute regurgitation: analysis of postoperative results from a multicentre study. Eur I Cardiothorac Surg 2008; 33: 573-82.

8. Bitner M, Maciejewski M. Mitral valve repair and coronary artery by-pass grafting in a case of ruptured posteromedial papillary muscle (muscle head reimplantation, artificial PTFE chords and ring implantation). Abstracts 24th EACTS Meeting. Interactive CardioVascular and Thoracic Surgery 2010; 11 (Suppl 2): 576

9. Vassileva CM, Boley T, Markwell S, et al. Meta-analysis of shortterm and long-term survival following repair versus replacement for ischemic mitral regurgitation. Eur J Cardiothorac Surg 2011; 39: 295-303.

10. Michalik M, Frask A, Trybull A, et al. Methods of treatment for gallbladder diseases. Videosurgery Miniinv 2009; 4: 121-5.

11. Bierca J, Kowalski B, Kosim A, et al. Lethal, iatrogenic complications of laparoscopic cholecystectomy. Videosurgery Miniinv 2006; 1: 10-2.

12. Frater $\mathrm{R}$, Vetter $\mathrm{H}$, Zussa $\mathrm{C}$, et al. Chordal replacement in mitral valve repair. Circulation 1990; 82 (Suppl IV): IV-125-30.

13. Aoyama T, Kawaguchi O, Teramoto T, et al. [Mitral valve replacement for mitral regurgitation caused by papillary muscle head rupture complicating acute myocardial infarction: report of a case]. Kyobu Geka 2005; 58: 239-42.

14. Shimokuni T, Yanagiya A, Okada W, et al. [Successful emergency surgery for acute mitral regurgitation due to total rupture in the anterior papillary muscle after acute myocardial infarction: report of a case]. Kyobu Geka 2008; 61: 495-9.

Received: 18.09.2012, revised: 25.10.2012, accepted: 12.11.2012. 